\title{
ERROR BOUNDS FOR QUASI-MONTE CARLO INTEGRATION WITH NETS
}

\author{
CHRISTIAN LÉCOT
}

\begin{abstract}
We analyze the error introduced by approximately calculating the $s$-dimensional Lebesgue measure of a Jordan-measurable subset of $I^{s}=[0,1)^{s}$. We give an upper bound for the error of a method using a $(t, m, s)$-net, which is a set with a very regular distribution behavior. When the subset of $I^{s}$ is defined by some function of bounded variation on $\bar{I}^{s-1}$, the error is estimated by means of the variation of the function and the discrepancy of the point set which is used. A sharper error bound is established when a $(t, m, s)$-net is used. Finally a lower bound of the error is given, for a method using a $(0, m, s)$-net. The special case of the 2-dimensional Hammersley point set is discussed.
\end{abstract}

\section{INTRODUCTION}

Applications of quasi-Monte Carlo methods arise in problems of numerical analysis that can be reduced to numerical integration. For $s \geq 2$ let $I^{s}=[0,1)^{s}$ be the half-open $s$-dimensional unit cube, and $\lambda_{s}$ be the $s$-dimensional Lebesgue measure. If $E$ is a Jordan-measurable subset of $I^{s}$ and $P$ is a set of $|P|$ points $\mathbf{x}_{1}, \ldots, \mathbf{x}_{|P|}$ evenly distributed over $I^{s}$, the volume of $E$ can be approximated by

$$
\frac{A(E, P)}{|P|}
$$

where $A(E, P)$ is the number of $p$ 's, $1 \leq p \leq|P|$, for which $\mathbf{x}_{p} \in E$. An analysis of the error

$$
\left|\frac{A(E, P)}{|P|}-\lambda_{s}(E)\right|
$$

was given in a paper of Niederreiter and Wills [5]. The error was bounded by means of $D(P)^{1 / s}$. The discrepancy $D(P)$ of the point set $P$ is defined by

$$
D(P)=\sup _{J}\left|\frac{A(J, P)}{|P|}-\lambda_{s}(J)\right|,
$$

Received by the editor October 10, 1994 and, in revised form, February 15, 1995.

1991 Mathematics Subject Classification. Primary 65C05; Secondary 11K38.

Key words and phrases. Quasi-Monte Carlo method, $(t, m, s)$-nets, discrepancy. 
where $J$ runs through all subintervals of $I^{s}$. We focus on special point sets $P$, which are called nets. The following definitions were given by Niederreiter [3]. Let $b \geq 2$ be an integer. An elementary interval in base $b$ is an interval of the form

$$
J=\prod_{i=1}^{s}\left[\frac{a_{i}}{b^{d_{i}}}, \frac{a_{i}+1}{b^{d_{i}}}\right)
$$

with integers $d_{i} \geq 0$ and integers $0 \leq a_{i}<b^{d_{i}}$ for $1 \leq i \leq s$. Let $0 \leq t \leq m$ be integers. A point set $P$ of $b^{m}$ points in $I^{s}$ is a $(t, m, s)$-net in base $b$ if $A(J, P)=b^{t}$ for every elementary interval $J$ in base $b$ with $\lambda_{s}(J)=b^{t-m}$. Discrepancy bounds for nets are given in the article of Niederreiter [3]. They can be used in conjunction with the estimation of Niederreiter and Wills to obtain error bounds. It is much simpler to estimate the error directly and an improved upper bound can be established.

The following case deserves special attention. Let $f: \bar{I}^{s-1} \rightarrow \bar{I}$ be a function of bounded variation in the sense of Hardy and Krause. We refer to the monograph of Niederreiter [4] for the definition of the concept of variation. Define the set

$$
E_{f}=\left\{\mathbf{x}=\left(\mathbf{x}^{\prime}, x_{s}\right) \in I^{s}: x_{s}<f\left(\mathbf{x}^{\prime}\right)\right\}
$$

For any set $P$ of $|P|$ points $\mathbf{x}_{1}, \ldots, \mathbf{x}_{|P|}$ in $I^{s}$, we have

$$
\left|\frac{A\left(E_{f}, P\right)}{|P|}-\lambda_{s}\left(E_{f}\right)\right|=\left|\frac{1}{|P|} \sum_{p=1}^{|P|} c_{E_{f}}\left(\mathbf{x}_{p}\right)-\int_{I^{s}} c_{E_{f}} d \lambda_{s}\right|,
$$

where $c_{E_{f}}$ is the characteristic function of $E_{f}$. The Koksma-Hlawka inequality (see below) cannot be used to derive an upper bound for the right-hand side of (2), because the variation of $c_{E_{f}}$ can be infinite for a very smooth $f$ ( let for instance $\left.f\left(\mathbf{x}^{\prime}\right)=x_{1}\right)$. In an earlier communication [1], we give an estimate of the left-hand side of (2) by means of the variation of $f$. The analysis used the estimation of Niederreiter and Wills. An improved upper bound is directly established in the present paper. In the case where $P$ is a $(t, m, s)$-net, there is a different method of proof which yields a better result.

Finally some lower bounds for the error (1) are established, which are not too dissimilar to the upper bounds. The analysis is restricted to $(0, m, s)$-nets and uses techniques of Schmidt [6]. For the 2-dimensional Hammersley point set (see [4]), a better result is given.

An outline of the paper is as follows. In $\S 1$ we derive an upper bound for the error (1) when the point set is a $(t, m, s)$-net in base $b$. In $\S 2$ we give an estimate of the left-hand side of (2). A smaller bound is given when $P$ is a net. In $\S 3$ we establish some lower bounds for the error (1) when $P$ is a $(0, m, s)$-net in base $b$.

\section{UPPER BOUNDS FOR NETS}

We recall the result of Niederreiter and Wills, when the original Euclidean norm is replaced by the maximum norm $\|\mathbf{x}\|_{\infty}=\max _{1 \leq i \leq s}\left|x_{i}\right|$. For $\varepsilon>0$ define

$$
\begin{aligned}
E_{\varepsilon} & =\left\{\mathbf{x} \in I^{s}: \exists \mathbf{y} \in E\|\mathbf{x}-\mathbf{y}\|_{\infty}<\varepsilon\right\}, \\
E_{-\varepsilon} & =\left\{\mathbf{x} \in I^{s} \quad: \forall \mathbf{y} \in I^{s} \backslash E\|\mathbf{x}-\mathbf{y}\|_{\infty} \geq \varepsilon\right\} .
\end{aligned}
$$


If there exists a positive nondecreasing function $\sigma$ defined for all $\varepsilon>0$ such that

$$
\forall \varepsilon>0 \quad \max \left(\lambda_{s}\left(E_{\varepsilon} \backslash E\right), \lambda_{s}\left(E \backslash E_{-\varepsilon}\right)\right) \leq \sigma(\varepsilon),
$$

then

$$
\left|\frac{A(E, P)}{|P|}-\lambda_{s}(E)\right| \leq 3 \sigma\left(\left\lfloor\frac{1}{D(P)^{1 / s}}\right\rfloor^{-1}\right)+D(P)^{1 / s} .
$$

If, in addition, $\left\{x_{s}:\left(\mathbf{x}^{\prime}, x_{s}\right) \in E\right\}$ is a subinterval of $I$ containing 0 , for every $\mathbf{x}^{\prime} \in I^{s-1}$, then

$$
\left|\frac{A(E, P)}{|P|}-\lambda_{s}(E)\right| \leq \sigma\left(\left\lfloor\frac{1}{D(P)^{1 / s}}\right\rfloor^{-1}\right)+D(P)^{1 / s} .
$$

Proposition 1. Let $E$ be a Jordan-measurable subset of $I^{s}$ and $P$ be a $(t, m, s)$-net in base $b$. If there exists a positive nondecreasing function $\sigma$ defined for all $\varepsilon>0$ such that

$$
\forall \varepsilon>0 \quad \max \left(\lambda_{s}\left(E_{\varepsilon} \backslash E\right), \lambda_{s}\left(E \backslash E_{-\varepsilon}\right)\right) \leq \sigma(\varepsilon),
$$

then

$$
\left|\frac{A(E, P)}{|P|}-\lambda_{s}(E)\right| \leq \sigma\left(b^{-\left\lfloor\frac{m-t}{s}\right\rfloor}\right)
$$

Proof. Let $d=\left\lfloor\frac{m-t}{s}\right\rfloor$. For $\mathbf{a}=\left(a_{1}, \ldots, a_{s}\right)$ with integers $a_{i}, 0 \leq a_{i}<b^{d}$, define $I_{\mathbf{a}}=\prod_{i=1}^{s}\left[\frac{a_{i}}{b^{d}}, \frac{a_{i}+1}{b^{d}}\right)$. Let

$$
E_{-}=\bigcup_{I_{\mathrm{a}} \subset E} I_{\mathrm{a}} \text { and } \quad E_{+}=\bigcup_{I_{\mathrm{a}} \cap E \neq \emptyset} I_{\mathrm{a}} .
$$

Then

$$
\begin{aligned}
& \frac{A\left(E_{-}, P\right)}{|P|}-\lambda_{s}\left(E_{-}\right)-\lambda_{s}\left(E \backslash E_{-}\right) \leq \frac{A(E, P)}{|P|}-\lambda_{s}(E), \\
& \frac{A(E, P)}{|P|}-\lambda_{s}(E) \leq \frac{A\left(E_{+}, P\right)}{|P|}-\lambda_{s}\left(E_{+}\right)+\lambda_{s}\left(E_{+} \backslash E\right) .
\end{aligned}
$$

The subsets $E_{-}$and $E_{+}$are disjoint unions of elementary intervals $I_{\mathbf{a}}$, with $\lambda_{s}\left(I_{\mathbf{a}}\right) \geq$ $b^{t-m}$, hence $\frac{A\left(E_{-}, P\right)}{|P|}-\lambda_{s}\left(E_{-}\right)=\frac{A\left(E_{+}, P\right)}{|P|}-\lambda_{s}\left(E_{+}\right)=0$. Furthermore, $E \backslash E_{-} \subset$ $E \backslash E_{-\frac{1}{b^{d}}}$, hence $\lambda_{s}\left(E \backslash E_{-}\right) \leq \sigma\left(\frac{1}{b^{d}}\right)$ and $E_{+} \backslash E \subset E_{\frac{1}{b^{d}}} \backslash E$, hence $\lambda_{s}\left(E_{+} \backslash E\right) \leq$ $\sigma\left(\frac{1}{b^{d}}\right)$.

\section{UPPER BOUNDS FOR SPECIAL SUBSETS}

Let $f: \bar{I}^{s-1} \rightarrow \bar{I}$ be a function of bounded variation $V(f)$. Then define $E_{f}$ by $E_{f}=\left\{\mathbf{x}=\left(\mathbf{x}^{\prime}, x_{s}\right) \in I^{s}: x_{s}<f\left(\mathbf{x}^{\prime}\right)\right\}$ and let $c_{E_{f}}$ be the characteristic function of $E_{f}$. If $P^{\prime}$ is a set of $\left|P^{\prime}\right|$ points $\mathbf{x}_{1}^{\prime}, \ldots, \mathbf{x}_{\left|P^{\prime}\right|}^{\prime}$ in $I^{s-1}$ and $P$ is a set of $|P|$ points $\mathbf{x}_{1}, \ldots, \mathbf{x}_{|P|}$ in $I^{s}$, the integral

$$
\int_{I^{s-1}} f d \lambda_{s-1}=\int_{I^{s}} c_{E_{f}} d \lambda_{s}
$$


can be approximated by

$$
\frac{1}{\left|P^{\prime}\right|} \sum_{p=1}^{\left|P^{\prime}\right|} f\left(\mathbf{x}_{p}^{\prime}\right) \quad \text { or by } \quad \frac{1}{|P|} \sum_{p=1}^{|P|} c_{E_{f}}\left(\mathbf{x}_{p}\right) .
$$

The second approximation, where the numerator is an integer, can be useful for numerical simulation (see $[1,2])$. In the first case, an error bound is given by the Koksma-Hlawka inequality

$$
\left|\frac{1}{\left|P^{\prime}\right|} \sum_{p=1}^{\left|P^{\prime}\right|} f\left(\mathbf{x}_{p}^{\prime}\right)-\int_{I^{s-1}} f d \lambda_{s-1}\right| \leq V(f) D^{\star}\left(P^{\prime}\right) .
$$

The star discrepancy $D^{\star}\left(P^{\prime}\right)$ of the point set $P^{\prime}$ is defined by

$$
D^{\star}\left(P^{\prime}\right)=\sup _{J}\left|\frac{A\left(J, P^{\prime}\right)}{\left|P^{\prime}\right|}-\lambda_{s-1}(J)\right|,
$$

where $J$ runs through all subintervals of $I^{s-1}$ containing 0 . In the second case, we have the following error estimate.

Proposition 2. Let $P$ be a point set of $|P|$ points in $I^{s}$ and $f: \bar{I}^{s-1} \rightarrow \bar{I}$ be of bounded variation in the sense of Hardy and Krause. If $D(P) \leq V(f)$, we have

$$
\left|\frac{A\left(E_{f}, P\right)}{|P|}-\lambda_{s}\left(E_{f}\right)\right| \leq s V(f)\left\lfloor\left(\frac{V(f)}{D(P)}\right)^{1 / s}\right\rfloor^{-1} .
$$

Proof. We shall prove somewhat better but more complicated estimates. Let $n_{1}, \ldots, n_{s-1}$ be integers. For $\mathbf{a}^{\prime}=\left(a_{1}, \ldots, a_{s-1}\right)$ with integers $a_{i}, 0 \leq a_{i}<n_{i}$, define $I_{\mathbf{a}^{\prime}}=\prod_{i=1}^{s-1}\left[\frac{a_{i}}{n_{i}}, \frac{a_{i}+1}{n_{i}}\right)$ and let

$$
\underline{E}=\bigcup_{\mathbf{a}^{\prime}} I_{\mathbf{a}^{\prime}} \times\left[0, \inf _{I_{\mathbf{a}^{\prime}}} f\right), \bar{E}=\bigcup_{\mathbf{a}^{\prime}} I_{\mathbf{a}^{\prime}} \times\left[0, \sup _{I_{\mathbf{a}^{\prime}}} f\right), F=\bigcup_{\mathbf{a}^{\prime}} I_{\mathbf{a}^{\prime}} \times\left[\inf _{I_{\mathbf{a}^{\prime}}} f, \sup _{I_{\mathbf{a}^{\prime}}} f\right] .
$$

Then

$$
\frac{A(\underline{E}, P)}{|P|}-\lambda_{s}(\underline{E})-\lambda_{s}(F) \leq \frac{A\left(E_{f}, P\right)}{|P|}-\lambda_{s}\left(E_{f}\right) \leq \frac{A(\bar{E}, P)}{|P|}-\lambda_{s}(\bar{E})+\lambda_{s}(F) .
$$

If $V(f) \leq D(P)$, choose $n_{1}=\cdots=n_{s-1}=1$. Then

$$
\left|\frac{A\left(E_{f}, P\right)}{|P|}-\lambda_{s}\left(E_{f}\right)\right| \leq D^{\star}(P)+\sup _{\bar{I}^{s-1}} f-\inf _{\bar{I}^{s-1}} f .
$$

Suppose $V(f) \geq D(P)$. The subsets $\underline{E}$ and $\bar{E}$ are disjoint unions of subintervals of $I^{s}$, hence

$$
\left|\frac{A(\underline{E}, P)}{|P|}-\lambda_{s}(\underline{E})\right| \leq D(P) \prod_{i=1}^{s-1} n_{i} \quad \text { and } \quad\left|\frac{A(\bar{E}, P)}{|P|}-\lambda_{s}(\bar{E})\right| \leq D(P) \prod_{i=1}^{s-1} n_{i} .
$$


We have $\lambda_{s}(F) \leq V(f) \sum_{i=1}^{s-1} \frac{1}{n_{i}}$ by Lemma 1 (see below). Therefore,

$$
\left|\frac{A\left(E_{f}, P\right)}{|P|}-\lambda_{s}\left(E_{f}\right)\right| \leq D(P) \prod_{i=1}^{s-1} n_{i}+V(f) \sum_{i=1}^{s-1} \frac{1}{n_{i}} .
$$

Put $x=\left(\frac{V(f)}{D(P)}\right)^{1 / s}$ and $r=\left\lfloor s \frac{\log x-\log \lfloor x\rfloor}{\log (\lfloor x\rfloor+1)-\log \lfloor x\rfloor}\right\rfloor-1$. If $r=-1$ or 0 , let $n_{1}=\cdots=$ $n_{s-1}=\lfloor x\rfloor$. Then

$$
\left|\frac{A\left(E_{f}, P\right)}{|P|}-\lambda_{s}\left(E_{f}\right)\right| \leq V(f)\left\lfloor\left(\frac{V(f)}{D(P)}\right)^{1 / s}\right\rfloor^{-1}\left(\left(\frac{\lfloor x\rfloor}{x}\right)^{s}+s-1\right),
$$

which yields the desired bound. If $0<r<s-1$, let $n_{i}=\lfloor x\rfloor+1$, for $1 \leq i \leq r$, and $n_{i}=\lfloor x\rfloor$, for $r<i \leq s-1$. Then

$$
\left|\frac{A\left(E_{f}, P\right)}{|P|}-\lambda_{s}\left(E_{f}\right)\right| \leq V(f)\left\lfloor\left(\frac{V(f)}{D(P)}\right)^{1 / s}\right\rfloor^{-1}\left(s-\frac{r+1}{\lfloor x\rfloor+1}\right),
$$

which yields the desired estimate.

Lemma 1. Let $f$ be a function of bounded variation on $\bar{I}^{s}$. Let $n_{1}, \ldots, n_{s}$ be integers. For $\mathbf{a}=\left(a_{1}, \ldots, a_{s}\right)$ with integers $a_{i}, 0 \leq a_{i}<n_{i}$, let $I_{\mathbf{a}}=\prod_{i=1}^{s}\left[\frac{a_{i}}{n_{i}}, \frac{a_{i}+1}{n_{i}}\right)$ and $\mathbf{y}_{\mathbf{a}}, \mathbf{z}_{\mathbf{a}} \in \bar{I}_{\mathbf{a}}$. Then

$$
\sum_{\mathbf{a}}\left|f\left(\mathbf{z}_{\mathbf{a}}\right)-f\left(\mathbf{y}_{\mathbf{a}}\right)\right| \leq V(f) \prod_{i=1}^{s} n_{i} \sum_{i=1}^{s} \frac{1}{n_{i}} .
$$

Proof. If $\mathbf{y}, \mathbf{z} \in \bar{I}^{s}$, let $T_{\mathbf{y}}^{(i)} f$ be the restriction of $f$ to the hyperplane $x_{i}=y_{i}$ and $\Delta_{(\mathbf{z}, \mathbf{y})}^{(i)} f=T_{\mathbf{z}}^{(i)} f-T_{\mathbf{y}}^{(i)} f$. If $K=\left\{i_{1}, \cdots, i_{k}\right\} \subset[1, s]$, we set

$$
T_{\mathbf{y}}^{K} f=T_{\mathbf{y}}^{\left(i_{1}\right)} \cdots T_{\mathbf{y}}^{\left(i_{k}\right)} f \quad \text { and } \quad \Delta_{(\mathbf{z}, \mathbf{y})}^{K} f=\Delta_{(\mathbf{z}, \mathbf{y})}^{\left(i_{1}\right)} \cdots \Delta_{(\mathbf{z}, \mathbf{y})}^{\left(i_{k}\right)} f .
$$

We put $T_{\mathbf{y}} f=T_{\mathbf{y}}^{[1, s]} f$. Let $\mathbf{1}=(1, \ldots, 1), \mathbf{a}+=\left(a_{1}+1, \ldots, a_{s}+1\right)$ and $\mathbf{x}_{\mathbf{a}}=$ $\left(\frac{a_{1}}{n_{1}}, \ldots, \frac{a_{s}}{n_{s}}\right)$. The desired estimate is established by combining the following identities:

$$
\begin{gathered}
f\left(\mathbf{z}_{\mathbf{a}}\right)-f\left(\mathbf{y}_{\mathbf{a}}\right)=\sum_{i=1}^{s} \Delta_{\left(\mathbf{z}_{\mathbf{a}}, \mathbf{y}_{\mathbf{a}}\right)}^{(i)} T_{\mathbf{y}_{\mathbf{a}}}^{[1, i-1]} T_{\mathbf{z}_{\mathbf{a}}}^{[i+1, s]} f, \\
T_{\mathbf{w}} f=\sum_{k=0}^{s}(-1)^{k} \sum_{\substack{K<[1, s] \\
\# K=k}} \Delta_{\left(\mathbf{x}_{\mathbf{a}+}, \mathbf{w}\right)}^{K} T_{\mathbf{x}_{\mathbf{a}+}}^{K^{c}} f, \quad \text { for } \quad \mathbf{w} \in \bar{I}_{\mathbf{a}} \quad \text { and } \\
T_{\mathbf{x}_{\mathbf{a}+}} f=\sum_{k=0}^{s}(-1)^{k} \sum_{\substack{K \subset[1, s] \\
\# K=k}} \sum_{\substack{a_{i}<b_{i}<n_{i} \\
i \in K}} \Delta_{\left(\mathbf{x}_{\mathbf{b}+}, \mathbf{x}_{\mathbf{b}}\right)}^{K} T_{\mathbf{1}}^{K^{c}} f . \quad \square
\end{gathered}
$$

When $P$ is a net, an improved estimate can be established. We shall use the following lemma of Niederreiter [3, Lemma 3.4.(ii)]. 
Lemma 2. Let $P$ be a $(t, m, s)$-net in base $b$. For any elementary interval $J^{\prime} \subset$ $I^{s-1}$ in base $b$ and for any $x_{s} \in \bar{I}$ one has

$$
\left|\frac{A\left(J^{\prime} \times\left[0, x_{s}\right), P\right)}{|P|}-\lambda_{s}\left(J^{\prime} \times\left[0, x_{s}\right)\right)\right| \leq b^{t-m} .
$$

Proposition 3. Let $P$ be $a(t, m, s)$-net in base $b$ and $f: \bar{I}^{s-1} \rightarrow \bar{I}$ be of bounded variation in the sense of Hardy and Krause. If $b^{t-m} \leq V(f)$, we have

$$
\left|\frac{A\left(E_{f}, P\right)}{|P|}-\lambda_{s}\left(E_{f}\right)\right| \leq s V(f) b^{-\left\lfloor\frac{m-t}{s}+\frac{\log V(f)}{s \log b}\right\rfloor} .
$$

Proof. Let $d_{1}, \ldots, d_{s-1}$ be integers and replace $n_{i}$ by $b^{d_{i}}$ in the proof of Proposition 2. If $V(f) \leq b^{t-m}$, choose $d_{1}=\cdots=d_{s-1}=0$. By Lemma 2, we have

$$
\left|\frac{A\left(E_{f}, P\right)}{|P|}-\lambda_{s}\left(E_{f}\right)\right| \leq b^{t-m}+\sup _{\bar{I}^{s-1}} f-\inf _{\bar{I}^{s-1}} f .
$$

Suppose $V(f) \geq b^{t-m}$. By Lemmas 1 and 2 , we obtain

$$
\left|\frac{A\left(E_{f}, P\right)}{|P|}-\lambda_{s}\left(E_{f}\right)\right| \leq b^{t-m+d_{1}+\cdots+d_{s-1}}+V(f) \sum_{i=1}^{s-1} \frac{1}{b^{d_{i}}} .
$$

Put $x=\frac{m-t}{s}+\frac{\log V(f)}{s \log b}$ and $r=\lfloor s x\rfloor-s\lfloor x\rfloor-1$. If $r=-1$ or 0 , let $d_{1}=\cdots=$ $d_{s-1}=\lfloor x\rfloor$. Then

$$
\left|\frac{A\left(E_{f}, P\right)}{|P|}-\lambda_{s}\left(E_{f}\right)\right| \leq V(f) b^{-\left\lfloor\frac{m-t}{s}+\frac{\log V(f)}{s \log b}\right\rfloor}\left(b^{s(\lfloor x\rfloor-x)}+s-1\right),
$$

which yields the desired estimate. If $0<r<s-1$, let $d_{i}=\lfloor x\rfloor+1$, for $1 \leq i \leq r$, and $d_{i}=\lfloor x\rfloor$, for $r<i \leq s-1$. Then

$$
\left|\frac{A\left(E_{f}, P\right)}{|P|}-\lambda_{s}\left(E_{f}\right)\right| \leq V(f) b^{-\left\lfloor\frac{m-t}{s}+\frac{\log V(f)}{s \log b}\right\rfloor}\left(b^{\lfloor s x\rfloor-s x-1}+s-r\left(1-\frac{1}{b}\right)-1\right),
$$

which yields the desired bound.

\section{LOWER BOUNDS FOR NETS}

We want to show that the order of magnitude of the bound in Proposition 1 is best possible. We shall establish a lower bound for the error (1). We refer to the lectures of Schmidt [6, Theorem 13.B] for a similar result. Since a $(0, m, s)$-net in base $b$ is also a $(t, m, s)$-net in base $b$, for any $t \leq m$, it follows that an interesting lower bound is only available for $t=0$. We recall the following result of Niederreiter [3, Corollary 5.11].

Lemma 3. For $m \geq 2 a(0, m, s)$-net in base $b$ can only exist if $s \leq b+1$.

If $x \geq 0$, let $\lceil x\rceil$ be the smallest integer $\geq x$. 
Proposition 4. Let $P$ be a $(0, m, s)$-net in base b. There exists a subset $E$ of $I^{s}$ with

$$
\forall \varepsilon>0 \quad \max \left(\lambda_{s}\left(E_{\varepsilon} \backslash E\right), \lambda_{s}\left(E \backslash E_{-\varepsilon}\right)\right) \leq\left(2^{s-1}+1\right) \varepsilon
$$

and

$$
\begin{aligned}
& \left|\frac{A(E, P)}{|P|}-\lambda_{s}(E)\right| \geq \frac{1}{4(s-1) ! b^{\frac{m}{s}}}, \quad \text { if s divides } m, \\
& \left|\frac{A(E, P)}{|P|}-\lambda_{s}(E)\right| \geq \frac{1}{4 b^{s-1+\left\lfloor\frac{m}{s}\right\rfloor}}, \quad \text { otherwise. }
\end{aligned}
$$

Proof. We shall prove sharper but more complicated bounds. Let $d_{1}, \ldots, d_{s}$ be integers with $d_{1}+\cdots+d_{s}=m$. For $\mathbf{a}^{\prime}=\left(a_{1}, \ldots, a_{s-1}\right)$ with integers $a_{i}, 0 \leq a_{i}<$ $b^{d_{i}}$, define $I_{\mathbf{a}^{\prime}}=\prod_{i=1}^{s-1}\left[\frac{a_{i}}{b^{d_{i}}}, \frac{a_{i}+1}{b^{d_{i}}}\right)$ and $a_{s}\left(\mathbf{a}^{\prime}\right)=a_{1}+\cdots+a_{s-1}$. Let

$$
\underline{\alpha}\left(\mathbf{a}^{\prime}\right)=\frac{1}{b^{d_{s}}}\left(a_{s}\left(\mathbf{a}^{\prime}\right)+\frac{1}{2} A\left(I_{\mathbf{a}^{\prime}} \times\left[\frac{a_{s}\left(\mathbf{a}^{\prime}\right)+1 / 2}{b^{d_{s}}}, \frac{a_{s}\left(\mathbf{a}^{\prime}\right)+1}{b^{d_{s}}}\right), P\right)\right),
$$

if $a_{s}\left(\mathbf{a}^{\prime}\right)<b^{d_{s}}$; otherwise $\underline{\alpha}\left(\mathbf{a}^{\prime}\right)=1$. Let $\bar{\alpha}\left(\mathbf{a}^{\prime}\right)=\underline{\alpha}\left(\mathbf{a}^{\prime}\right)+\frac{1}{2 b^{d_{s}}}$, if $a_{s}\left(\mathbf{a}^{\prime}\right)<b^{d_{s}}$; otherwise $\bar{\alpha}\left(\mathbf{a}^{\prime}\right)=1$. Let

$$
\underline{E}=\bigcup_{\mathbf{a}^{\prime}} I_{\mathbf{a}^{\prime}} \times\left[0, \underline{\alpha}\left(\mathbf{a}^{\prime}\right)\right), \quad \bar{E}=\bigcup_{\mathbf{a}^{\prime}} I_{\mathbf{a}^{\prime}} \times\left[0, \bar{\alpha}\left(\mathbf{a}^{\prime}\right)\right) .
$$

Let $\varepsilon>0$. We have $\underline{E}_{\varepsilon} \backslash \underline{E} \subset \underline{F}^{(\varepsilon)} \cup \underline{G}^{(\varepsilon)}$, where

$$
\begin{gathered}
\underline{F}^{(\varepsilon)}=\bigcup_{\mathbf{a}^{\prime}} I_{\mathbf{a}^{\prime}} \times\left[\underline{\alpha}\left(\mathbf{a}^{\prime}\right), \underline{\alpha}\left(\mathbf{a}^{\prime}\right)+\varepsilon\right), \\
\underline{G}^{(\varepsilon)}=\bigcup_{i=1}^{s-1} \bigcup_{\mathbf{a}^{\prime}} \prod_{j=1}^{i-1}\left[\frac{a_{j}}{b^{d_{j}}}, \frac{a_{j}+1}{b^{d_{j}}}\right) \times\left(\frac{a_{i}}{b^{d_{i}}}-\varepsilon, \frac{a_{i}}{b^{d_{i}}}\right) \times \prod_{j=i+1}^{s-1}\left(\frac{a_{j}}{b^{d_{j}}}-\varepsilon, \frac{a_{j}+1}{b^{d_{j}}}\right) \\
\times\left[\underline{\alpha}\left(\mathbf{a}_{-i}^{\prime}\right)+\varepsilon, \underline{\alpha}\left(\mathbf{a}^{\prime}\right)+\varepsilon\right), \\
\mathbf{a}_{-i}^{\prime}=\left(a_{1}, \ldots, a_{i-1}, a_{i}-1, a_{i+1}, \ldots, a_{s-1}\right) .
\end{gathered}
$$

Furthermore,

$$
\underline{E_{\varepsilon}} \backslash \underline{E} \subset \bigcup_{\mathbf{a}^{\prime}} I_{\mathbf{a}^{\prime}} \times\left[\underline{\alpha}\left(\mathbf{a}^{\prime}\right), \underline{\alpha}\left(\mathbf{a}^{\prime}\right)+\frac{1}{b^{d_{s}}}\left(\sum_{i=1}^{s-1}\left\lceil\varepsilon b^{d_{i}}\right\rceil+\frac{1}{2}\right)+\varepsilon\right) .
$$

On the other hand, we have $\underline{E} \backslash \underline{E}_{-\varepsilon} \subset \underline{F}^{(-\varepsilon)} \cup \underline{G}^{(-\varepsilon)}$, where

$$
\begin{gathered}
\underline{F}^{(-\varepsilon)}=\bigcup_{\underline{\alpha}\left(\mathbf{a}^{\prime}\right)<1} I_{\mathbf{a}^{\prime}} \times\left(\underline{\alpha}\left(\mathbf{a}^{\prime}\right)-\varepsilon, \underline{\alpha}\left(\mathbf{a}^{\prime}\right)\right), \\
\underline{G}^{(-\varepsilon)}=\bigcup_{i=1}^{s-1} \bigcup_{\underline{\alpha}\left(\mathbf{a}^{\prime}\right)<1} \prod_{j=1}^{i-1}\left[\frac{a_{j}}{b^{d_{j}}}, \frac{a_{j}+1}{b^{d_{j}}}\right) \times\left[\frac{a_{i}+1}{b^{d_{i}}}, \frac{a_{i}+1}{b^{d_{i}}}+\varepsilon\right) \\
\times \prod_{j=i+1}^{s-1}\left[\frac{a_{j}}{b^{d_{j}}}, \frac{a_{j}+1}{b^{d_{j}}}+\varepsilon\right) \times\left(\underline{\alpha}\left(\mathbf{a}^{\prime}\right)-\varepsilon, \underline{\beta}\left(\mathbf{a}_{+i}^{\prime}\right)\right], \\
\mathbf{a}_{+i}^{\prime}=\left(a_{1}, \ldots, a_{i-1}, a_{i}+1, a_{i+1}, \ldots, a_{s-1}\right), \\
\underline{\beta}\left(\mathbf{a}^{\prime}\right)=\underline{\alpha}\left(\mathbf{a}^{\prime}\right)-\varepsilon \quad \text { if } \underline{\alpha}\left(\mathbf{a}^{\prime}\right)<1 ; \text { otherwise } \underline{\beta}\left(\mathbf{a}^{\prime}\right)=1 .
\end{gathered}
$$


Furthermore,

$$
\underline{E} \backslash \underline{E}_{-\varepsilon} \subset \bigcup_{\mathbf{a}^{\prime}} I_{\mathbf{a}^{\prime}} \times\left(\underline{\alpha}\left(\mathbf{a}^{\prime}\right)-\frac{1}{b^{d_{s}}}\left(\sum_{i=1}^{s-1}\left\lceil\varepsilon b^{d_{i}}\right\rceil+\frac{1}{2}\right)-\varepsilon, \underline{\alpha}\left(\mathbf{a}^{\prime}\right)\right) .
$$

Similar results are available for $\bar{E}$. By the definition of $\underline{E}$ and $\bar{E}$, we have

$$
\begin{gathered}
\left|\frac{A(\underline{E}, P)}{|P|}-\lambda_{s}(\underline{E})\right|+\left|\frac{A(\bar{E}, P)}{|P|}-\lambda_{s}(\bar{E})\right| \\
=\frac{1}{2 b^{m}} \#\left\{\mathbf{a}^{\prime} \in \mathbf{N}^{s-1}: 0 \leq a_{i}<b^{d_{i}} \text { for } 1 \leq i<s \text { and } a_{s}\left(\mathbf{a}^{\prime}\right)<b^{d_{s}}\right\} .
\end{gathered}
$$

If $s$ divides $m$, let $d_{1}=\cdots=d_{s}=d=\frac{m}{s}$. Then

$$
\forall \varepsilon>0 \quad \max \left(\lambda_{s}\left(\underline{E}_{\varepsilon} \backslash \underline{E}\right), \lambda_{s}\left(\underline{E} \backslash \underline{E}_{-\varepsilon}\right), \lambda_{s}\left(\bar{E}_{\varepsilon} \backslash \bar{E}\right), \lambda_{s}\left(\bar{E} \backslash \bar{E}_{-\varepsilon}\right)\right) \leq \sigma(\varepsilon),
$$

where

$$
\sigma(\varepsilon)=\min \left(\frac{\left(\begin{array}{c}
b^{d}+s-2 \\
s-1
\end{array}\right) \varepsilon}{b^{(s-1) d}}+\frac{\left(\begin{array}{c}
b^{d}+s-3 \\
s-2
\end{array}\right)\left(\left(1+\varepsilon b^{d}\right)^{s-1}-1\right)}{b^{(s-1) d}}, \varepsilon+\frac{(s-1)\left\lceil\varepsilon b^{d}\right\rceil+1 / 2}{b^{d}}\right) .
$$

On the other hand, we have

$$
\max \left(\left|\frac{A(\underline{E}, P)}{|P|}-\lambda_{s}(\underline{E})\right|,\left|\frac{A(\bar{E}, P)}{|P|}-\lambda_{s}(\bar{E})\right|\right) \geq \frac{\left(\begin{array}{c}
b^{d}+s-2 \\
s-1
\end{array}\right)}{4 b^{m}},
$$

which yields the desired result. If $s$ does not divide $m$, let $d_{1}=\cdots=d_{s-1}=d=$ $\left\lfloor\frac{m}{s}\right\rfloor$ and $d_{s}=m-(s-1) d$. By Lemma 3 , we have

$$
\forall \varepsilon>0 \quad \max \left(\lambda_{s}\left(\underline{E}_{\varepsilon} \backslash \underline{E}\right), \lambda_{s}\left(\underline{E} \backslash \underline{E}_{-\varepsilon}\right), \lambda_{s}\left(\bar{E}_{\varepsilon} \backslash \bar{E}\right), \lambda_{s}\left(\bar{E} \backslash \bar{E}_{-\varepsilon}\right)\right) \leq \widetilde{\sigma}(\varepsilon),
$$

where

$$
\widetilde{\sigma}(\varepsilon)=\min \left(\varepsilon+\frac{\left(1+\varepsilon b^{d}\right)^{s-1}-1}{b^{d}}, \varepsilon+\frac{(s-1)\left\lceil\varepsilon b^{d}\right\rceil+1 / 2}{b^{d_{s}}}\right) .
$$

On the other hand, we have

$$
\max \left(\left|\frac{A(\underline{E}, P)}{|P|}-\lambda_{s}(\underline{E})\right|,\left|\frac{A(\bar{E}, P)}{|P|}-\lambda_{s}(\bar{E})\right|\right) \geq \frac{b^{(s-1) d}}{4 b^{m}},
$$

which yields the desired result.

In the 2-dimensional case, a sharper estimate is available for the Hammersley point set in base $b$,

$$
P=\left\{\left(p b^{-m}, \phi_{b}(p)\right): 0 \leq p<b^{m}\right\},
$$

where $\phi_{b}$ is the radical inverse function in base $b$ (see [4]). It is easily seen that $P$ is a $(0, m, 2)$-net in base $b$. 
Proposition 5. Let $P$ be the two-dimensional Hammersley point set in base $b$ and let $T=\left\{\left(x_{1}, x_{2}\right) \in I^{2}: x_{2}<x_{1}\right\}$. Then

$$
\forall \varepsilon>0 \quad \max \left(\lambda_{2}\left(T_{\varepsilon} \backslash T\right), \lambda_{2}\left(T \backslash T_{-\varepsilon}\right)\right) \leq 2 \varepsilon
$$

and

$$
\left|\frac{A(T, P)}{|P|}-\lambda_{2}(T)\right|=\frac{1}{2 b^{\left\lfloor\frac{m}{2}\right\rfloor}} .
$$

Proof. A point $\left(x_{1}, x_{2}\right) \in P$ if and only if $\left(x_{2}, x_{1}\right) \in P$. Moreover, $b^{\left\lfloor\frac{m+1}{2}\right\rfloor}$ points of $P$ lie on the line $x_{1}=x_{2}$. Hence the result of the proposition follows.

Beside the class of $(t, m, s)$-nets, a class of sequences, called $(t, s)$-sequences was introduced by Niederreiter [3]. By his techniques, error estimates for $(t, s)$-sequences can be obtained from error estimates for $(t, m, s)$-nets.

\section{BIBLIOGRAPHY}

1. C. Lécot, A quasi-Monte Carlo method for the Boltzmann equation, Math. Comp. 56 (1991), 621-644. MR 91j:65008

2. W. J. Morokoff and R. E. Caflisch, A quasi-Monte Carlo approach to particle simulation of the heat equation, SIAM J. Numer. Anal. 30 (1993), 1558-1573. MR 94k:65009

3. H. Niederreiter, Point sets and sequences with small discrepancy, Monatsh. Math. 104 (1987), 273-337. MR 89c: 11120

4. $\_$Random number generation and quasi-Monte Carlo methods, SIAM, Philadelphia, PA, 1992. MR 93h:65008

5. H. Niederreiter and J. M. Wills, Diskrepanz und Distanz von Maßen bezüglich konvexer und Jordanscher Mengen, Math. Z. 144 (1975), 125-134; Berichtigung, ibid. 148 (1976), 99. MR 51:12763; MR 53:7996

6. W. M. Schmidt, Lectures on irregularities of distribution, Tata Institute of Fundamental Research, Bombay, 1977. MR 81d:10047

Laboratoire de Mathématiques, Université de Savoie, 73376 Le Bourget du Lac, FRANCE

E-mail address: lecot@univ-savoie.fr 